Mots. Les langages du politique

\title{
(Web)campagne présidentielle. L'incertaine influence des nouvelles formes de médiatisation
}

Presidential election (web)campaign. The uncertain influence of the new forms

of mediatization

(Web)campaña presidencial. La incierta influencia de las nuevas formas de mediatización

\section{Jean-Michel Rampon}

\section{OpenEdition}

\section{Journals}

Édition électronique

URL : https://journals.openedition.org/mots/22442

DOI : $10.4000 /$ mots. 22442

ISSN : 1960-6001

Éditeur

ENS Éditions

\section{Édition imprimée}

Date de publication : 15 octobre 2016

Pagination : 53-60

ISBN : 978-2-84788-850-8

ISSN : 0243-6450

\section{Référence électronique}

Jean-Michel Rampon, «(Web)campagne présidentielle. L'incertaine influence des nouvelles formes de médiatisation », Mots. Les langages du politique [En ligne], 112 | 2016, mis en ligne le 15 octobre 2018, consulté le 22 avril 2022. URL : http://journals.openedition.org/mots/22442 ; DOI : https://doi.org/ $10.4000 /$ mots. 22442 


\section{(Web)campagne présidentielle. L'incertaine influence des nouvelles formes de médiatisation}

Ce court article, qui ne prétend pas à l'exhaustivité quant aux références convoquées, voudrait bien plutôt interroger une tendance lourde que l'on a vue se confirmer lors de la précédente campagne présidentielle en France, quand bien même l'évolution était déjà en germe dès celle de 2007, avec les ÉtatsUnis d'Amérique comme inspirateurs (Serfaty, 2009) : le renforcement d'une forme de communication politique apparue dans ses contours contemporains dès l'élection de 2004 outre-Atlantique, caractérisée par une polyphonie énonciative qui se double désormais d'un brouillage accru quant aux instances de parole en jeu. Pour le dire autrement, si le blog est venu perturber le système de médiatisation qui prévalait jusqu'alors lors d'une élection présidentielle, le tweet comme dispositif technique n'a fait qu'ajouter à la métamorphose d'un «écosystème » formé des acteurs politiques, des producteurs d'information et de leurs publics (Tumasjan et al., 2010).

Il va s'agir ici de présenter quelques-uns des enjeux globaux en lien avec la diversité accrue des outils ou extensions créés pour ce média spécifique qu'est Internet, avec toujours la télévision comme pôle central, quand bien même prend-elle des formes nouvelles et engage-t-elle des formats nouveaux ${ }^{1}$. Seront d'abord présentés quelques éléments de mise en perspective de la médiatisation en termes d'influence, avant de sérier quelques-unes des conséquences quant aux positionnements énonciatifs des acteurs concernés, non sans s'arrêter parallèlement sur la nomination de ces formes émergentes de communication politico-médiatique autant que sur celle, inédite, des individus ou groupes qui y prennent part.

1. À l'occasion de la session du «Club de l'économie » du quotidien Le Monde du 20 janvier 2016 («http://www.lemonde.fr/le-club-de-l-economie/article/2016/01/21/delphine-ernotte-laconcurrence-aujourd-hui-ce-sont-les-plates-formes-numeriques_4851484_4795074.html>, consulté le 2 juin 2016), la présidente de France Télévisions Delphine Ernotte a affirmé que le terme télévision change, que ce type de média ne représente plus une succession choisie de programmes regardés de manière linéaire et que les plateformes numériques comme Netflix, HBO ou Amazon seront dans un délai proche les premiers concurrents des télévisions en France, ce qui n'est pas sans poser un problème de modèle économique autant que démocratique.

Université de Lyon, Sciences Po-Lyon, ELICO jean-michel.rampon@sciencespo-lyon.fr 


\section{Médiatisation et influence des «leaders d'opinion »}

«Sortis vainqueur de leur bataille avec les journalistes pour la maîtrise de la médiatisation, les communicants devront donc sans doute trouver de nouvelles stratégies face à l'émergence de ce social network turn qui risque fort de modifier le schéma du two-step-flow of communication et le jeu de la persuasion politique, en introduisant de nouveaux influenceurs dans le jeu et en modifiant les conditions de réception critique du discours politique médiatisé » (Mercier, 2013, p. 200). Ainsi s’achève le chapitre d'un livre consacré à la «communication politique bien singulière » à l'œuvre lors de la dernière élection présidentielle française de 2012 . On y retrouve la notion de médiatisation, la référence au couple singulier journaliste-communicant et l'évocation de la «théorie de la communication à double étage», par laquelle Paul Lazarsfeld a pointé le rôle médiateur de l'influence interpersonnelle dans le processus de persuasion de masse ${ }^{2}$, y compris dans un contexte d'élections présidentielles (Lazarsfeld et al., 1944). Ce modèle, un temps négligé, a repris de la vigueur chez certains auteurs, à l'instar d'Elisabeth Noelle-Neumann. Après avoir mis l'accent sur le rôle des «leaders d'opinion » de la télévision et de la radio dans l'instauration d'une opinion dominante poussant la majorité à s'inscrire dans une «spirale du silence», cette auteure a par la suite privilégié une approche du leader d'opinion plus en accord avec une conception lazarsfeldienne de l'individu meneur, que suivent d'autres individus bien plus nombreux (Maigret, 2015, p. 202-203).

Pour Gilles Bastin, la conception de la politique en campagne comme une activité «médiatisée» productrice d'effets en matière informationnelle va de pair avec une montée en puissance de l'audience de la télévision. À tel point que cet auteur a pu repérer que le terme même s'impose en pleine période de généralisation des médias télévisuels de masse. Au risque de vider de son contenu les principes du modèle lazarsfeldien : «D'un côté, l'analyse a été en partie désociologisée (les mass media audiovisuels étant réputés avoir une audience de masse et toucher toutes les catégories sociales, les variables traditionnelles d'analyse du comportement électoral sont moins pertinentes); de l'autre les campagnes électorales reprennent leur importance et deviennent l'objet d'analyses et l'enjeu de débats comme des “campagnes médiatiques" » (Bastin, 2007, p. 3). Ce dernier énoncé n'est pas sans faire écho à celui qui clôt le passage suivant d'un article de l'analyste du discours Dominique Maingueneau à l'occasion des trente ans de la revue Mots. Les langages du politique : «Le renforcement de l'emprise de la télévision, puis le développement fulgurant d'Internet ont considérablement modifié les conditions de l'énonciation

2. Voir ma mise en perspective (Rampon, 2011). 
politique et les relations entre le discours politique et les citoyens », cette transformation ayant eu pour effet de produire « un brouillage de la frontière entre les études sur les médias et les études sur le discours politique (le fameux duel qui clôt la campagne présidentielle est à la fois un genre politique et un genre télévisuel) »(Maingueneau, 2010, p. 88). Il convient donc de fournir en deuxième partie de ce propos quelques éléments d'appréciation quant aux positionnements énonciatifs, sinon rhétoriques, induits par de tels changements, jusque dans le rôle accru dévolu à une parole qui n'a pas toujours été présentée comme autorisée.

\section{Information en ligne et coproductions rédactionnelles}

Pour Marc Lits, «l'analyse des usages d'Internet pose clairement la question de l'éclatement énonciatif qu'il installe» et «c'est dans la construction et l'identification des positions énonciatives que se joue probablement un nouveau dispositif de communication » (Lits, 2009, p. 97). Les expressions crossmedia ou transmedia sont à cet égard révélatrices d'une telle fragmentation, encore qu'elles éclairent mal le phénomène de montée en puissance d'une parole plus profane. Tandis qu'avec le crossmedia, un même récit de base se trouve décliné sur différents supports, avec le transmedia, ce sont au contraire ces derniers qui, considérés séparément, vont « raconter une bribe de récit appartenant à l'univers narratif global» (Degand, Grevisse, 2012, p. 328), à l'instar d'une campagne électorale.

La diversité induite par la multiplication de sites dédiés à l'information sur Internet ne va donc pas de soi. Si le Web peut aller de pair avec une «disparité des modes de traitement journalistiques» autant que des "énonciations éditoriales» (Touboul, Damian-Gaillard, Marty, 2012, à partir du cas d'un sondage sur Marine Le Pen et la présidentielle de 2012), il peut être aussi le lieu où repérer des formes variées de reprise de l'information en ligne, quel que soit par ailleurs son profil, du site natif (Mediapart) au média en ligne (Le Monde), en passant par l'infomédiaire (MSN Actu). Sur la base de la «webcampagne» de l'élection présidentielle de 2012 comme sujet et source pour les journalistes, Simon Gadras a mis en valeur plusieurs niveaux de redondance de l'information en ligne, de la moins à la plus marquée, comme lors d'un événement sur lequel la focale est ponctuellement posée. Par rapport à l'usage des contenus disponibles sur les «espaces web» des candidats, l'auteur conclut que «la mise en scène de la distanciation journalistique ne passe pas tant par le commentaire ou l'analyse critique que par la mobilisation d'éléments factuels explicitement issus des sources, en particulier des données chiffrées» (Gadras, 2016). Soit probablement une manière d'être toujours tributaire, sinon prisonnière, d'un cadrage de la campagne sur la base du registre de la compétition. 
Les contenus médiatiques produits le sont donc de moins en moins par des utilisateurs aux tâches auctoriales circonscrites, ce qui n'est pas sans effet sur les pratiques journalistiques, qui se retrouvent en l'espèce dé-routinisées. Que l'on pense à des types de coproduction rédactionnelle qui, lors de l'élection présidentielle française de 2012 , se sont démarqués d'une couverture ou d'un traitement médiatique classique. Encore que ce recours aux internautes peut prendre des atours variés et avoir une influence différente sur/dans le milieu des journalistes selon que ces derniers sont orienteurs (1) ou orientés (2), autrement dit dans la position ici de devoir suivre les internautes et leurs commentaires plutôt que de leur proposer par exemple des pistes de compréhension de l'enjeu électoral ou de contrôle de la parole proférée par ceux et celles qui aspirent à la plus haute fonction élective :

1. Soit l'exemple du Véritomètre (aujourd'hui disparu), mis en place sur le site d'information www.owni.fr "pour déceler le vrai, le faux et l'imprécis au sein d'une multitude d'interventions des candidats à la présidentielle de 2012 " avec l'aide d'internautes qui «ont, à cette occasion, prêté main-forte aux journalistes dans la vérification des chiffres et des déclarations » (Degand, Grevisse, 2012, p. 111). Bien que cet «approvisionnement par la foule» (crowdsourcing) puisse être sujet à caution - que vaut un chiffre correcteur sans prise en compte des modalités ayant gouverné son apparition? Une telle “externalisation" des tâches n'appauvrit-elle pas l'entreprise de presse qui s'y adonne, quel que soit son modèle, ainsi que les fournisseurs de contenu « ordinaires», peu ou mal rémunérés ? etc. -, il va de pair avec l'émergence d'une information que l'on ne peut plus présenter comme spécifiquement journalistique. Certes, la production médiatique se caractérise par des paroles et des sources qui lui sont extérieures et dont elle rend compte, de façon plus ou moins explicite ou plus ou moins codée, dans sa matérialité langagière même. Mais dans le cas présent, ce mixage s'opère dès l'enveloppe du média où il est pour ainsi dire revendiqué comme tel, ce qui n'est pas anodin dans un événement ritualisé comme l'élection présidentielle et ses rendez-vous coutumiers.

2. Soit la référence aux « ripostes-parties», d'abord propres au champ politique avant que d'émigrer vers le champ médiatique, ou plus exactement propices à s'étendre en raison de la présence d'un grand nombre de journalistes, en particulier d'information politique, sur les réseaux sociaux3. La bataille des tweets - ou «tweet-clash» - sert d'abord aux partis politiques français en période électorale à montrer aux électeurs, indépendamment des médias qui restent malgré tout une courroie possible de transmission, leur capacité à combattre l'adversaire sur le terrain des idées, mais aussi à faire mieux que

3. Simon Gadras (2016) définit les « ripostes parties» comme des « rassemblements sur Twitter de sympathisants des candidats, en particulier à l'occasion des principales interventions télévisées de leur candidat, organisées par les équipes de campagne et ayant pour objectif de diffuser un maximum de messages favorables au candidat». 
lui en matière de prédictions et de programme. On peut inférer à partir de là que c'est la présence avérée des journalistes sur les réseaux sociaux qui va amener le phénomène à être fortement médiatisé et à toucher un plus grand public (Theviot, 2014, p. 96). Plus exactement, les ripostes parties, d'opérations politiques «publicisables» depuis le monde politique, deviennent dans un deuxième temps des opérations proprement médiatiques, en particulier à l'heure des meetings en direct, voire des télévisions ou radios propres aux partis, "afin de proposer aux journalistes des supports visuels pour réaliser des reportages», quand une telle médiatisation n'est pas synonyme de modification du format éditorial des programmes politiques télévisés, avec insertion de hashtags spécifiques au gré des émissions (Theviot, 2014, p. 101 et 104).

\section{Télévision connectée et « twiléspectateurs "}

La «télévision 2.0 » renvoie au surcroît d'interaction permis par la télévision dite connectée dans le cadre de certains de ses programmes (dont les débats d'avant élection), au point qu'Arnaud Mercier est allé jusqu'à créer un néologisme, twiléspectateurs, lui servant à désigner «ces citoyens qui, en direct, commentent et/ou suivent les commentaires sur Twitter d'une prestation politique télévisée» (Mercier, 2013, p. 169). Pour autant, il ne faut pas perdre de vue qu'une telle activité sur et via les réseaux sociaux, si l'on veut bien suspendre un temps la référence aux «professionnels » de la politique et des médias, n'est le fait que d'une minorité d'acteurs, les plus enclins à soutenir une cause ou à défendre des idées contre un ou plusieurs adversaires, mais aussi à savoir utiliser les outils numériques dans leur déclinaison la plus contemporaine. La description suivante rend assez bien compte du processus en jeu: «En termes rhétoriques, on peut donc dire que le locuteur n'est plus le seul responsable de la construction de son image. Cette responsabilité est dorénavant partagée avec l'auditoire. Il ne s'agit pas simplement de l'image que les électeurs se font du candidat [...], mais de l'image qu'ils font de lui » (Yanoshevsky, 2009, p. 66. C'est l'auteure qui souligne). Cela équivaut à dire que l'image des candidates et des candidats, longtemps chasse gardée des appareils politiques entendus dans une extension large qui englobe les conseillers en communication, est désormais aussi (et de plus en plus?) le produit d'une interrelation d'un nouveau genre.

Pour autant, de la même façon que le blog avant lui en période électorale, le tweet n'a pas pris ses aises tout seul. La montée en puissance des chaînes d'information en continu, demandeuses autant que pourvoyeuses de nouvelles qu'il s'agit de renouveler le plus fréquemment possible au risque sinon de lasser le téléspectateur, en a facilité l'émergence et le recours par des voix qui ont tout intérêt à collaborer (le « twitto » journaliste en ce qu'il peut obtenir jusqu'à 
des informations inédites, quand bien même ne seraient-elles que de faible portée; le « twitto » citoyen ou cybermilitant en ce qu'il peut gagner en visibilité médiatique autant que réticulaire et en légitimité au sein même du cercle ou du parti depuis lequel il s'exprime). Par-delà, on se trouve face à un «écosystème » d'un nouveau genre, avec la montée en puissance de nouveaux dispositifs technico-médiatiques, où se trouvent imbriqués des discours, parfois à l'état de bribes, d'acteurs-locuteurs qui s'auto-(entre)tiennent les uns les autres. Par-delà les sphères en jeu, jusqu'à celle du foyer où le regard est potentiellement moins passif dans le cadre d'une télévision de plus en plus connectée, c'est aussi la question de la temporalité de l'information qui se trouve posée, à l'instar du direct des meetings dont le commentaire se poursuit après la fin, en quelque sorte rendu public sur le même support télévisuel qui en aura assuré la diffusion. Une manière de densifier l'événement et son traitement ou de permettre ce que la télévision, par son seul canal historique, n'aura pas été capable d'assouvir?

Comme l'écrit Isabelle Veyrat-Masson au moment de présenter une recherche collective ayant porté sur l'élection présidentielle de 2007, l'étude de la médiatisation de la campagne électorale a pu être menée «sous l'angle des trois principaux acteurs du triangle de la communication politique : d'une part, les candidats et le peuple mis en scène dans les médias, d'autre part, les vecteurs eux-mêmes du message politique, les médias, à travers leur fonction de gatekeeping, de sélection des nouvelles et, enfin, les récepteurs de la campagne électorale médiatisée, les électeurs» (Veyrat-Masson, 2011, p.13). Si la deuxième partie du livre produit à partir d'une telle recherche porte sur les médias (la télévision certes, mais aussi la presse écrite, la radio et Internet), un début de fossé s'opère avec une autre recherche multi-laboratoires plus récente, portant quant à elle sur l'élection présidentielle suivante, celle de 2012 (Gerstlé, Magni Berton, 2014). Cette fois de «nouveaux» outils de communication, formes renouvelées de médiat(isat)ion que le réseau des réseaux rend possible, y sont davantage représentés, ce qui témoigne de leur place croissante dans la passation et le partage de contenus dans un espace public en transformation. Cette évolution d'ores et déjà avalisée atteindra-t-elle un seuil lors de la prochaine élection présidentielle en France, ou ses effets s'en verront-ils accentués?

Au-delà de ce que ces formes renouvelées peuvent entraîner, il est probable que se perpétue un phénomène déjà identifié par Éric Maigret : «À mesure que l'on se rapproche de l'élection, les partis et les candidats retrouvent en fait des trajectoires assez connues, la dynamique politique semble par conséquent bien plus ancrée dans les différences sociales que dans la seule circulation de l'information» (Maigret, p. 204-205). Ce qui doit excéder un tel constat, c'est qu'en l'absence de délimitation claire de ce qu'est la démocratie, il convient d'inclure dans ce qui peut la renouveler « les revendications micropolitiques et toutes les constructions réflexives et identitaires liées aux pro- 
grammes des médias de masse, sans opposer systématiquement nouveaux et anciens médias [...], sans les opposer aussi aux pratiques parlementaires » (Ibid., p. 291). Tandis qu'une récente étude relève l'influence sur les résultats électoraux du média singulier qu'est Google en fonction du rang obtenu par les candidats dans le classement produit après requête (Epstein, Robertson, 2015), il convient d'en restreindre la portée tant il est possible aux internautes encore indécis de s'informer à d'autres sources, y compris numériques - en continuité en quelque sorte avec ce qui a pu être observé pour les «anciens» médias, où la causalité simple n'est guère de mise en raison d'un approvisionnement dans des agendas diversifiés (politiques, médiatiques et citoyens) et en constante interaction.

\section{Références}

BAStin Gilles, 2007, «Médiatisation(s) et codage de l'événement en régime d'élection. Premiers résultats d'une enquête empirique sur les espaces de médiatisation de la campagne présidentielle de 2007 ", communication au Congrès de l'AFSP, Toulouse, septembre 2007, 〈 https://halshs.archives-ouvertes.fr/halshs-00332841〉, document PDF, 11 p. (consulté le 2 juin 2016).

Degand Amandine, Grevisse Benoît éd., 2012, Journalisme en ligne. Pratiques et recherches, Bruxelles, De Boeck.

EPSTEIN Robert, ROBERTSON Ronald E., 2015, «The search engine manipulation effect (seme) and its possible impact on the outcomes of elections", Proceedings of the National Academy of Sciences of the USA, vol.112, n033, 〈http://www.pnas.org/ content/112/33/E4512.abstract> (consulté le 2 juin 2016).

GADRAS Simon, 2016 (à paraître), «Circulation et redondance de l'information en ligne. La webcampagne de l'élection présidentielle française de 2012 comme sujet et source pour les journalistes», Informer avec internet. Reprises et métamorphoses de l'information, I. Hare, J.-M. Rampon, J.-F. Tétu, A. Touboul éd., Besançon, Presses universitaires de Franche-Comté.

Gerstlé Jacques, MAgni Berton Raul éd., 2014, 2012, la campagne présidentielle. Observer les médias, les électeurs, les candidats, Paris, Pepper, L'Harmattan.

LazArsfeld Paul F., Berelson Bernard, Gaudet Hazel, 1944, The People's Choice. How the Voter Makes up his Mind in a Presidential Campaign, New York, Columbia University Press.

LITS Marc, 2009, «La médiatisation du politique ou le passage d'un espace public délibératif à un espace public symbolique narratif », A contrario, vol. 2, nº12, p. 85-100.

MAIGRET Éric, 2015, Sociologie de la communication et des médias, Paris, Armand Colin, $3^{\mathrm{e}}$ édition.

Maingueneau Dominique, 2010, "Le discours politique et son "environnement" ", Mots. Les langages du politique, n94, "Trente ans d'étude des langages du politique (1980-2010)», p. 85-90. 
MerCier Arnaud, 2013, "Avènement du Twiléspectateur et hashtags contestataires, faits marquants de la campagne sur les réseaux sociaux», Présidentielle 2012. Une communication politique bien singulière, P. J. Maarek éd., Paris, L'Harmattan, p. 165-200.

RAMPoN Jean-Michel, 2011, "Elihu Katz et Paul L. Lazarsfeld (1955/2008), Influence personnelle. Ce que les gens font des médias, Paris, Armand Colin / Institut national de l'audiovisuel», Communication, vol.29, $\mathrm{n}^{\circ} 1$, 〈http://communication.revues. org/2550> (consulté le 2 juin 2016).

Serfaty Viviane, 2009, "Présidentielles aux États-Unis. La communication politique au prisme de l'internet (1996-2008) », Questions de communication, nº ${ }^{0}$, p. 367382.

THeVIOT Anaïs, 2014, " “Twitter en regardant la télé” : une campagne transmédias interactive? Analyse comparée des stratégies numériques du Parti socialiste et de l'Union pour un mouvement populaire lors des ripostes-party », Télévision, vol.1, $n^{0} 5$, p. 95-111.

Touboul Annelise, DAmian-GaIlLard Béatrice, MARTY Emmanuel, 2012, «La disparité des modes de traitement journalistiques et des énonciations éditoriales sur le web. Le cas d'un sondage sur Marine Le Pen et la Présidentielle de 2012 », Réseaux. Communication, technologie, société, no176, p. 73-103.

Tumasjan Andranik, Sprenger Timm, SAndner Philipp, WelPe Isabell, 2010, «Predicting elections with Twitter. What 140 characters reveal about political sentiment», Proceedings of the Fourth International AAAl Conference on Weblogs and Social Media, Menlo Park, The AAAI Press, p. 178-185.

VeYRAT-MASSON Isabelle éd., 2011, Médias et élections. La campagne présidentielle de 2007 et sa réception, Paris, l'Harmattan.

YANOSHEVSKY Galia, 2009, "L'usage des vidéoblogs dans l'élection présidentielle de 2007. Vers une image plurigérée des candidats", Mots. Les langages du politique, n89, « 2007. Débats pour l’Élysée », p. 57-68. 